\title{
Principles for Tertiary-Level Astronomy Courses
}

\author{
Derek McNally \\ Department of Physical Sciences, University of Hertfordshire, College \\ Lane, Hatfield, Herts., AL109AB, U.K. e-mail: dmn@star.herts.ac.uk
}

\begin{abstract}
Any worthwhile tertiary-level course of study should, as its highest priority, reflect the discipline it represents as it is contemporaneously practiced. Were it not to do so, students would be intellectually underprovided. This paper sets out general principles which a first degree-level course in astronomy should aim to provide for its students. No specific syllabi will be attempted but, rather, the paper will outline ranges of topics and their level of treatment. While all students taking such courses should have as professional experience as possible, it must be recognised that most students taking tertiary-level astronomy courses may not become professional astronomers and that such courses will necessarily have to have flexibility to meet local circumstances.
\end{abstract}

\section{Introduction}

On a global basis, the objectives of tertiary education (education beyond school, in particular university education) are legion. Local, national needs and requirements, educational tradition, expectation of tertiary education and availability of resources will all, in varying proportions, shape the content of tertiary-education courses. Consequently little point will be served in setting out detailed syllabi for a universal astronomy/astrophysics degree course. However, astronomy is an eclectic discipline - it is one of the purest of sciences, being concerned with the study of matters having apparently little connection with day-to-day life. On the other hand astronomy is very much an applied science drawing together other sciences - chemistry, physics, biology, while drawing heavily on engineering skills and the whole being heavily influenced by mathematics. Astronomy is therefore a marvelous vehicle to extend horizons and to generate awareness of the interconnectedness of scientific endeavour. Astronomy is also pushing at the boundaries of scientific knowledge - the frisson of the possibility for fundamental discovery is always present. And astronomy - however eclectic - is a useful science of great value in the affairs of mankind. Sadly, astronomers have, in the past century, been reluctant to push the educational value of their science and have abdicated science teaching very largely to others. The final decades of the twentieth century have seen renewed growth in astronomy teaching - often in the form of add-on and optional courses in other science-degree courses. The 
value of astronomy as science education, because of its applied nature, is something that awaits exploitation and, together with other similar sciences, has the potential to reinvigorate interest in the physical sciences.

There are three major pathways in which astronomy is currently used in tertiary education:

i science for those not studying a physical science discipline;

ii courses with another degree such as physics or mathematics;

iii degree courses specialising in astronomy.

The first pathway has been exploited in many countries - in particular the United States - and has had the benefit of extensive course material and textbook development. A criticism of the pathway is that it does not develop a feeling for the disciplines of the physical/mathematical sciences for the very good reason that the undergraduates taking such courses lack an adequate physicalsciences/mathematics background. This pathway, while it spreads awareness and limited understanding of astronomy, maybe even generating a lifetime interest, has allowed a lacuna to develop between the first and third pathways which the second does not fulfil - namely courses in physical science for those adequately qualified but who may not find a specialist course attractive and turn, for their tertiary education, to other disciplines. This is a serious neglect since the twenty-first century will need a large cadre of people with a broad scientific education, appreciating the inter-relatedness of the sciences and having sufficient depth of knowledge to understand the processes, the value and the limitation of scientific endeavor. The second pathway does not contribute to bridging that gap since the astronomy is often restricted to one course in one year of study and is not a sustained study over the full degree course.

Pathway (iii) - the specialist degree course on which I will concentrate - can take a variety of forms. It can concentrate on astrophysics, combine astrometry and astrophysics, be directed towards detector systems with the mix weighted to taste. To give a local example, the original astronomy degree at University College London had no component of cosmology as the then professor of astronomy considered cosmology equated with speculation. He attached overwhelming importance to evidence, obtaining it, critically evaluating it and, where considered sound, to using it. It is such that gives variation and colour to different degree courses. There are those who consider that astronomy degrees should be astrophysics degrees and should not contain anything on the astronomy of position, determination of time or celestial mechanics. Clearly vast advances have been made in astrophysics which enhance its attractiveness but, post-HIPPARCOS, such omissions may ignore astrophysically valuable techniques.

There is an interesting, very small, but persistent, group of undergraduates who are passionately devoted to celestial mechanics and the astronomy of position who find astrophysics tedious and can feel let down even by an "astronomy" degree course. Consequently in designing an astronomy degree one must consider carefully the motivation of prospective undergraduates and ensure that the range of such degrees is as wide as resources can sustain.

In this paper I shall look at the basic entry knowledge an undergraduate should possess on entry to an astronomy degree in section 2; the fundamental 
structure of astronomy will be addressed in section 3 and the development of that structure will be sketched in section 4 . I will look at the role of the practical and the project in degree-level work in section 5 before finally looking again in section 6 at some of the themes for the future of astronomy degrees.

\section{Pre-Entry Knowledge}

A person contemplating a degree in astronomy must recognise that it is a degree which demands a sound understanding of mathematics and physics. From this there can be no escape. The prospective undergraduate must therefore prepare by attempting to reach the highest levels of competence in both mathematics and physics that are offered by the secondary education system. There should be competence in the use of algebra, coordinate geometry and calculus. There is considerable value in experience of pure mathematics as well as applied. The student should have an understanding of mechanics, light and optics, electricity and magnetism and some introduction to basic atomic and nuclear physics. They should also have had considerable experience in laboratory physics. There has been a trend away from laboratory experience at all levels of education. Laboratories are expensive to establish and maintain; laboratory work is also expensive in time available in crowded curricula. This is probably one of the major weaknesses in science teaching since it is only by investigation that a student can develop any appreciation of why some scientific concepts at first seem to lack contact with the real world. Experiment also introduces concepts of error of measurement and brings in some element of computation. Laboratory work may indicate that theoretical studies may be a more productive route through tertiary education!

Clearly the basic background possessed by a student on entering tertiary education must be reinforced, developed and extended at the tertiary level. No degree course in astronomy can omit the development of understanding of physics and mathematics in its undergraduates. Indeed studies of physics and mathematics may take up to $50 \%$ of the course loading, particularly in the earlier years at university.

\section{Basic Elements of Astronomy}

Astronomy depends on the reception and interpretation of electromagnetic signals for the bulk of its information on the universe. Other forms of information carrier exist such as study of meteoritic bodies which impact the Earth, direct investigation of other solar-system bodies, detection of neutrinos and oof particles from cosmic rays. These last are important sources of information but are highly specialised in specific areas. For the purpose of this section, I will address only information that comes via electromagnetic radiation.

Gravity is a dominant force throughout astronomy from the orbits of planets and their satellites, through the structure and evolution of stars to the large scale structure of the universe. Accordingly therefore, undergraduate teaching must contain at least basic Newtonian dynamics and for advanced studies an introduction to the Special and General Theories of Relativity. 
An understanding of electromagnetism and gravitation are essential for a study of astrophysics but for a full appreciation of astronomy there must also be the additional element of the astronomy of position and the measurement of time. This area of astronomy has become seriously undervalued with the result that there is a declining number of astronomers with a good understanding of what positional astronomy can do in an astrophysical context. This gives cause for deep concern, given the valuable information in the HIPPARCOS catalogues and the prospects in the foreseeable future for microarcsecond astrometry. It can be argued that positional astronomy is unnecessary for the successful prosecution of astrophysical research - and there is an element of validity in such arguments given the very wide canvas of astrophysics. However, positional astronomy and measures of time should remain an important part of astronomy degrees - perhaps defining the essential difference between a degree in astronomy and a degree in astrophysics. Celestial mechanics is an interesting case in point in this connection. Celestial mechanics has been recalled from obscurity by the demands of space science. It has become extremely sophisticated. It does not have wide appeal and it is by no means unequivocally clear to me whether it should be presented as the highly mathematical end of astronomy teaching or form a part of applied mathematics. That it should remain available is essential even though it is never going to appeal to more than a handful of dedicated (often awesomely so) undergraduates.

\subsection{Electromagnetic radiation}

Any student of astronomy has to be aware of the emission, transmission and detection of electromagnetic radiation. Fundamental physics of reflection, refraction and polarisation should be understood at the level of application of Maxwell's Equations. Emission and absorption of radiation by atoms andappropriate atomic/quantum physics. Photoelectric phenomena should be addressed before developing the theory of emission, transmission and detection of electromagnetic radiation.

Emission of electromagnetic radiation An important aspect of emission of electromagnetic radiation, now often neglected, is a proper treatment of black-body radiation. Black bodies are best approximated under astronomical conditions where also departures from thermal equilibrium are clearly apparent. The black body relates emitted radiation to other properties of matter, e.g. temperature. The physics of black-body radiation is also an interesting study in thermodynamics and quantum statistics. An appreciation of black body radiation can often give a zero order approximation to a real situation and allow understanding of the essence of a situation which otherwise demands very difficult analysis - as with most problems encountered in the study of stellar atmospheres.

Emission of electromagnetic radiation also extends into line emission by atoms, ions and molecules and the relevant branches of atomic quantum physics. In the radio astronomy of today, the fine and hyperfine structure of energy levels of atoms and molecules are of considerable importance in the detection of interstellar species, for example.

Transmission of electromagnetic radiation The transmission of electromagnetic radiation through gases whether stellar, interstellar or intergalactic is of 
crucial astrophysical importance. In passing through matter, electromagnetic radiation can be absorbed, re-emitted, scattered and polarised. The consequences of that absorption, re-emission, scattering and polarisation can be detected and quantified with important consequences for understanding the physical state of the matter that produced those effects.

Again the opacity of stellar material is determined by the absorption and scattering properties of the constituents of stars. Opacity plays the dominating role in determining the structure of stars and that opacity is determined by the quantum properties of the atoms, ions and molecules which comprise the material of stars. It is crucial that any degree course in astronomy has (at least) a basic course on radiative transfer. Such a course will be the foundation for a wide variety of highly disparate advanced topics. No astronomer/astrophysicist can be without an appreciation of radiative transfer.

Detection of electromagnetic radiation Unless the radiation from cosmic objects can be detected and measured, there is little knowledge available from these objects. Again all astronomy undergraduates should be aware of how electromagnetic radiation can be acquired (basic telescope design), significant signal detected and how such signals are calibrated. This is not the same as a course on instrumentation which is highly dependent on frequencies observed and specific detector technologies. All undergraduates should be aware of how photons are collected, aware of such matters as resolution and signal strength. They should know that signals can be differentiated by frequency (e.g. spectroscopy) or that a number of frequencies can be integrated to enhance detector response and they should know about the available and projected detector systems of their generation. The detection of electromagnetic radiation lends itself to both the taught course and practical/observational/laboratory work. It is in this context that undergraduates should learn the all-important consequence of signal-to-noise ratio and the trade-offs that they will have to make in acquiring useful observations for themselves. Signal-to-noise ratio leads directly to error - a topic that is rarely touched on within the context of school science. Observational error is not a criticism but a fact of life. Adroitly used course work and practical work based on the detection of electromagnetic radiation can be a singularly useful way to engender good scientific practice.

The type of physics involved here is at some remove from mainstream physics teaching and is one reason why there should be specialist degrees in astronomy and astrophysics.

\subsection{Gravity}

Gravity is an associating force. On the scale of astrophysical enquiry it is the force which keeps planets in orbit around the Sun, maintains the Sun as a stable star and plays a key role in the dynamics of galaxies. Basic gravitational concepts appear in both school and university physics. One can therefore expect undergraduates to understand gravitation up to the extent of central-force elliptical orbits and Kepler's laws of planetary motion.

However, this is not the stage at which gravitation becomes astronomically useful. Orbits have to be related to path on the sky. Parabolic and hyperbolic orbits need introduction. The concept that six elements are required to define 
an orbit needs to be introduced together with elementary concepts on how such elements are to be determined (i.e. the interface to celestial mechanics) and the information that can be determined from objects such as binary stars when it is not always possible to determine all six orbital elements. The value of measuring radial velocities in place of spatial dimensions also needs to be introduced.

The concept of gravitational potentials needs to be developed in order to examine the equilibrium of stars. This leads to an important integrating study - stellar structure. Like a course on radiative transfer, so too should any degree course in astronomy have at a minimum, an introductory course on stellar structure. Stellar structure brings together many physical strands - gravitational forces, dynamics, energy transfer by radiation, energy generation by nuclear processes, thermodynamics, quantum physics. While modern stellar-structure theory is sophisticated and dependent on high-powered computers and computational methods, the fundamental aspects of stellar structure are readily demonstrable in a simple way. A mass-luminosity-radius relation can be derived in a straightforward way that demonstrates unequivocally the dependence of structure in stars on atomic physics and the evolution of stars driven by nuclear physics. Again the basis of fundamental techniques used in constructing stellar models can also be made clear in a simple way. A basic stellar-structure course can therefore establish criteria on which to come to grips with the sophisticated stellar models that are now being investigated and builds up basic understanding of the subject.

Astronomy and astrophysics degree courses have a basis of understanding of gravitation on which to build. It is important, however, to extend orbital concepts so that direct connection can be made to astrophysical technique actually in use in deriving astrophysical data from observations via gravitation. Stellar structure is one area of astrophysics where gravity plays an important direct role, in interaction with other physical process, to produce a highly sophisticated understanding of the internal structure of stars and their evolution which can be related, in turn, to relatively straightforward observations of the outer layers of stars.

\subsection{Positional astronomy and the measurement of time}

Sadly, positional astronomy and the measurement of time have become the poor relations in astronomical education. Because positional astronomy reached a peak of development towards the end of the nineteenthth century and because it required very painstaking work to produce a small progressive step, the blossoming of astrophysics in the early twentieth century displaced positional astronomy from its prime position as an astronomical activity. The success of the HIPPARCOS project should help to bring positional astronomy back towards the cutting edge but to be successful, there will have to be greater emphasis on positional astronomy courses within astronomy degree structures. It is here that a possible distinction might be drawn between astronomy and astrophysics degrees in that the latter need not pursue positional astronomy beyond that needed to locate objects. However, if positional astronomy is to be fully exploited as an astrophysical tool, then there must be good understanding of the nature of that tool. 
Positional astronomy, post-HIPPARCOS, now offers a quality of data on stellar distances, possible stellar multiplicity etc. that has hitherto been unavailable. That information is beginning to make an impact on the refinement of certain astrophysical parameters. A proper appreciation of these data may have, as yet unforeseen, revolutionary consequences. It is therefore important that facility in the use of the concepts of positional astronomy should be widely developed so that the full potential of the HIPPARCOS data-set can be exploited and appropriate astrophysical input generated to guide the planning of microarcsecond positional astronomy projects now being considered.

It is an even sadder reflection of the state of physical science in our time that the basis of time-measurement is so little understood within the physical sciences. In mitigation it could be argued that the procedure of timekeeping has become so refined, that accurate time services can be taken for granted the measurement of time is a Cinderella subject area because of its exceptional success. Yet as a global society we are critically dependent on the provision of accurate time services in every aspect of daily life. If there is one place where reference to the science of time-measurement should find a prime place, it is in the astronomy degree. Time-measurement may seem obscure and difficult in the search for precise definition that can be implemented practically. This has led to leap years, leap seconds, ephemeris time and all manner of pitfalls for the unwary. At present, there is a strong lobby in the world of satellite-based communication and navigation-service providers to get rid of leap seconds. There are few people with the necessary expertise and knowledge to argue that question or indeed critically assess the long term benefits/losses that might flow from a change of present timekeeping and management practices. Revolutionary changes are likely to occur in the near future on the formulation of celestial reference-frames and time dissemination. The astronomical community, more than any other, should acknowledge its responsibilities and ensure that astronomy degrees maintain adequate teaching on the measurement of time. It is acknowledged that this may not be the most electrifying experience for the average undergraduate but it does not have to be the dull grind it often is.

There is still a powerful necessity to maintain positional astronomy and measurement of time as a key part of basic astronomy degree level teaching - for the benefit of astrophysics as much as for retaining a body of people knowledgeable in this area for the purpose of maintaining proper public policy in accurate timekeeping.

\section{Gilding the Lily}

In the previous section I have looked at three key areas in tertiary astronomical education. Once these fundamental issues have been addressed the range of topics to be offered are legion. The world is indeed your oyster. Degrees can be developed around range of topics related to the specialist knowledge of the staff available to teach. In my view degree courses should be built on the premise that it is better to inculcate understanding in depth rather than see superficial comprehensive coverage.

Clearly any degree course is going to start with some form of introductory course describing in simple, straightforward terms, the entire range of the astro- 
nomical canvas from the Earth to the latest in cosmological thinking. However, it is unlikely that within the limitation of staff expertise and sheer limitation of time and resources available, that a satisfying comprehensive degree course in astronomy could be developed giving adequate depth to all components. Each degree will have to decide its principal focus, the range of departure from that focus, the type of student it wants to attract and the scientific value and capability of the graduate it will ultimately deliver to the world of work. Not all of these aspects pull in the same direction by any means and significant compromise will be needed to achieve scientific integrity and coherence within the framework of staff abilities and interests, university regulations and availability of resources. However, the ultimate criteria against which an astronomy degree should be judged will be its scientific integrity and coherence.

A degree should be further judged against its choice of scientific focus. For example a degree in astronomy might be focussed on planetary studies. But planetary studies cannot be properly pursued without good knowledge of the structure of the Earth and atmospheric physics. Nor can planetary studies be focussed without good knowledge of the structure and evolution of the Sun (and by implication the nature of stars other than the Sun). Such a degree might not necessarily offer an advanced course on the structure and evolution of the universe!

Some astronomy degrees should retain a place for celestial mechanics. The basis for celestial mechanics is very much focussed in advanced applied mathematics and numerical methods. However, celestial mechanics does have practical significance in the development of space navigation and should, as such, find a place in degree level astronomy teaching. The effect is twofold - the first to encourage interested students to consider celestial mechanics as a possible path towards a career but also to ensure that the expertise of celestial mechanics for space flows back into astronomical developments.

Celestial mechanics is a special case but there are many other special cases and every astronomy degree should offer at least one really advanced course which is offered in few other places worldwide - even if only relatively few students actually take it. Rarity disciplines are endangered species but they must not be allowed to become extinct. Sadly, the exigencies of modern economics may ensure such extinctions.

\section{Practical Astronomy}

It is often taken as axiomatic that practical work is such a good thing, that there is little need to justify its inclusion in a degree course and even in association with non-degree taught courses. After all says the mantra, access to the sky is freely available to all. That is so, but it is free, at best, only as far as the sixth visual magnitude. Thereafter the costs rise dramatically. Astronomy practical classes are costly to provide. Since a degree course should be aiming at professional standards and at inculcating a professional approach, then the equipment used in such practical courses must be at a professional standard, particularly for advanced classes. This means that an optical telescope should be sought at about $0.5-\mathrm{m}$ aperture and radio telescopes at about 2-3 $\mathrm{m}$. Since most telescopes for undergraduate teaching will be located in brightly lit and often radio noisy 
urban areas, there is not very much point seeking larger telescopes unless there are other reasons, e.g. a parallel research use, a specially favoured site. Any telescopes should have focal-plane arrangements which offer the strength and space to take relatively heavy detectors. The mountings for such telescopes should be stable and as free from locally generated vibration as is possible. Such provision will generate expense - particularly in respect of building works. Optical telescopes should be housed in domes (or equivalent structures) as a protection against wind buffeting. It should always be borne in mind that a carefully selected optical telescope, for example, mounted in an adequate dome can be written off on a period of 25 years, if not longer. An investment of $£ 500,000$ can therefore equate to an annual investment of $£ 20,000$ over a 25 year period.

Focal-plane instrumentation changes rapidly but, for example, good CCD cameras are highly affordable and can make excellent use of a $0.5-\mathrm{m}$ aperture optical telescope. It is important to be able to provide imaging, photometric and spectroscopic capability, i.e. to maximise the range of types of observation that can be attempted.

Clearly the level of provision in the early years of a degree course need incur less expense but even here there should be considerable flexibility for focal-plane instruments. It is a good maxim to buy the best possible telescope that can be afforded - both in respect to the quality of the optics and in respect of the construction of the mounting and drive. Good engineering is never cheap.

Unfortunately the costs of maintaining undergraduate laboratories in the physical sciences is high - they demand floor space, services, specialised equipment and adequate levels of staffing - both technical and teaching. In astronomy, optical-wavelength practicals are done at night, so the staffing levels must be high in order to ensure student safety while working in dark conditions. It should be borne in mind that radio astronomy and optical solar astronomy can be carried out during standard university working hours.

The positive gain from laboratory work is that students are forced to assess their capabilities, the capabilities of experimental systems, to recognise the omnipresence of error and, particularly in astronomy, to learn the value of proper preparation - a unique observational opportunity may be lost because of poor preparation just as much as through the vagaries of the weather. Laboratory work allows informal contact with teaching and research staff and thereby an opportunity for students to assess, and reassess, their future career plans. Practical work is a highly valuable pedagogical tool and it should have a high priority - sadly this is not always the case in our financially hard-pressed universities.

Project work is an important part of degree work. If adroitly used, projects can contribute greatly to the scientific maturation of undergraduates. On the other hand, projects can be difficult to assess, in that the contribution of individual students to a project may be hard to separate clearly from the contribution of peers and academic supervisors. A well considered assessment scheme can normally cope with such problems. Projects can take many forms - from an individual driven project, an academic-supervisor driven project to a group project comprising several different undergraduates. Projects need not be observational (in temperate climates, weather can be a considerable inhibitor of optical/IR observational projects though the advent of well-sited robotic telescopes could 
remove much of this problem) and can be as varied as the resources of the teaching department can permit. A limiting factor can often be the range of experience within the academic supervisors.

I tend to favour individual undergraduate projects as this builds up individual creativity (but at the expense of loss of experience of group working) though it must be recognised that individual projects are demanding of supervisory capability. Providing individual projects for a year of 20 students is demanding because self-driven undergraduates are relatively rare (and as such require little supervision but may need steering to maintain realism) and the necessary supervision needs the backing of a substantial research group. Projects are clearly for advanced students - but small projects can be incorporated within the body of practical teaching in all years to habituate students to this form of study.

Finally it is well worth considering observational field trips. One of the difficulties with the academic calendar is that classes must be scheduled - observational classes being no exception. If the local climate is not of the best, cloudy nights can apparently show an unhealthy correlation with scheduled classes. Field trips to a climatically well-found observatory can allow a week devoted exclusively to observational work from sunset to sunrise. Such work is particularly valuable in turning creative undergraduates into professional astronomers - the change can often be dramatic. Field trips are not expensive but not easy to arrange - few well-found observatories welcome the advent of undergraduates taking up valuable telescope time. The actual experience of real observing at a high-quality site is the essence of such trips - this cannot be obtained by using a robotic telescope to the same degree. Undergraduate field trips should be developed more widely in view of the beneficial personal development that they engender.

\section{The Future}

In this paper I have set out an outline of what a degree course in astronomy/astrophysics might contain. I have argued for certain basic elements in section 3 and advocated that a significant amount of course time should be devoted to practical/project work in section 5. I purposely did not set out a full syllabus in section 4 since it is clear that degrees can take many forms with different emphases meeting local needs, interests and expertise. To some, what I have argued for may seem unduly elitist and expensive. I do not believe it is elitist to provide a specialist degree and to treat that degree in a highly professional way. While many individual students taking astronomy degrees may never become professional astronomers, it would be utterly wrong to deny them, as undergraduates, a proper professional training. A professional approach is a highly portable skill, even if learnt within the confines of one particular science.

The criticism of expense is more justified. To set up and to maintain an astronomy degree is expensive - in terms of specialist staff, library provision and, in particular, in terms of observational provision. However, one can only operate within the income one has and that income should buy the best - in any area - that can be afforded. In many cases it may not be financially possible to offer a specialist astronomy/astrophysics degree. 
As I have argued elsewhere (McNally, 1998), it may be that the heyday of the specialist degree in the physical sciences is coming to an end. It may even be that the specialist degree, so good at reproducing our own kind, may no longer be the method of choice in developing a scientifically literate population. Why then spend on specialist degrees? Simply, to maintain a cadre of well-educated creative specialised physical scientists without whom the physical sciences will not develop. Nevertheless, numbers of undergraduates in the physical sciences may well continue to fall. Specialist-degree courses will remain but the specialisms will have to find new ways to generate income. This, I believe, will have to come through the development of widely based physical-science degrees, incorporating several specialist subjects, designed to produce knowledgeable scientists though not necessarily specialised scientists. Such courses will need to have wide appeal to generate sufficient (in the sense of meeting national needs) numbers of graduates. Astronomy should be a major player in the promotion and development of such degrees aimed at lifting the level of understanding science from its present abysmal levels (as is only too plain from the treatment of science by the media). The twenty-first century will be even more crucially affected by scientific advances than was the twentieth - to cope with these advances as a global society, it is imperative that we have a substantial number of people within the community who are not only scientifically literate, but literate and knowledgeable in the physical sciences - an area where undergraduates are still voting with their feet - if only to attempt to resolve the environmental challenges of the twentyfirst century in a rational manner. Such more widely based degrees in physical science will have to coexist with, and complement, the specialist degrees - and vice versa. The physical sciences are just too important to allow them to wither into a minority interest. Physical sciences such as astronomy will be of great importance in recruiting and retaining the interest of undergraduates studying selected groups of physical sciences. Astronomy should therefore assume a high profile in developing the teaching of the physical sciences in the twenty-first century - if we fail to produce proper physical science teaching then it is very likely that our response to current and future environmental challenges will be more likely to be disastrous than successful. That is the challenge which cannot be shirked.

\section{References}

McNally, D., 1998, University Education in the Next Century, New Trends in Astronomy Teaching, IAU Colloquium 162, Eds. Gouguenheim, L., McNally, D. and Percy, J.R., CUP, Cambridge, pp. 12-15.

\section{Discussion}

Bhatia asked if students who took pure astronomy were as employable as those who took some astronomy as part of physics. McNally replied that some of his students became astronomers, others found positions as mathemtaicians or computer scientists. 